\title{
Dynamical aspects of mean field theories for electrolytes and applications
}

\author{
Arik Yochelis ${ }^{1,2, a}$ and Katharina Krischer ${ }^{3, b}$ \\ ${ }^{1}$ Department of Solar Energy and Environmental Physics, Blaustein Institutes for \\ Desert Research (BIDR), Ben-Gurion University of the Negev, Sede Boqer Campus, \\ Midreshet Ben-Gurion 8499000, Israel \\ 2 Department of Physics, Ben-Gurion University of the Negev, Be'er Sheva 8410501, \\ Israel \\ ${ }^{3}$ Physik-Department E19a, Technische Universität München, James-Franck-Straße 1, \\ 85748 Garching, Germany
}

Received 11 February 2019

Published online 17 April 2019

Electrolytes are intriguing physicochemical compositions and soft matter/active media due to their combination of short- and long-range interactions. Moreover, they are at the heart of many applications that exploit their unique properties for specific purposes ranging from energy conversion to functional material designs, such as solar cells, batteries, supercapacitors, thin-film deposition methods, lubrication, fuel cells, synthesis and self-assembly of colloids, photo-electrochemical catalysis, and gas discharge plasmas.

While the basic principles of charge transfer and mass transport in electrolytes are outlined in many textbooks, modern applications demand careful revisions as the standard theories evolve about low concentrations of (point size) ions in either polar or apolar solvents at large volumes, i.e., concentrations lower than about 0.1 molar. As many aspects of these physicochemical systems imply specific charge transfer and transport properties, advances in these fields are largely driven by either atomistictype computations, such as density functional theory (DFT) and molecular dynamics (MD), or statistical mechanics descriptions about near steady-state conditions, e.g., modified Poisson-Boltzmann frameworks. Hence, most experimental observations and thus, applications, require also extended and detailed temporal insights for characterization, design, and stability, which are often beyond the scope of near steady-state approaches. Specifically, time-dependent mean field methods are required to delineate the basic mechanisms that should outline and help to advance realistic models in due course along with interpretation of experimental results to advance and improve devices.

This topical issue is designed to collect a broad range of studies devoted to spatiotemporal mean field methods that address both state of the art theories and application-driven ideas to electrolytes in polar and apolar solvents. The studies range from sophisticated thermodynamical treatments of electrolytic solutions, over state-of-the-art modeling of the electrical double layer in complex settings and electrokinetic systems to nanofluidics, nonlinear responses of electrochemical systems,

a e-mail: yochelis@bgu.ac.il

b e-mail: krischer@tum.de 
and the impact of spatial heterogeneities. In fact, there are many examples where at least two of these aspects cooperate.

In an electro-thermodynamical study, Dreyer et al. [1] model mobility coefficients and other electrolyte properties in the case of non-dilute electrolytes. Werkhoven et al. [2] present an analytical study on an electrokinetic system with a charged surface, and point out how different physical processes govern time and length scales. Matse et al. [3] consider electrokinetic transport in nanofluidic channels by focusing on Onsager's reciprocity principle for the case of rigid and deformable channel walls. The ionic flux through membranes in the presence of large permanent charges, as discussed by Zhang et al. [4], presents another advance to a theoretical framework at confined geometries. Bier and Yochelis [5] show that account of finite size effects allows to determine the width of the electrified interface and properties of steady state transient currents when spontaneously charged colloids are placed in apolar media. Different aspects of understanding and interpretation of nonlinear behaviors are considered in [6-8]. Wolff et al. [6] demonstrate that nonlinear frequency response analysis (NFRA) is a versatile tool to obtain insight on complex signatures governing the nonlinear dynamics of electrochemical cells. Using a modeling approach, Salman et al. uncover the origin of the negative differential resistance in the prototypical electrochemical oscillator involving anodic polarization of a semiconducting electrode [7]. Liu and Kiss [8] investigate localized dynamic features resulting from nonlinear kinetics combined with dual electrode flow cells, which render the effective parameters of the cell heterogeneous. Non-uniformities in the electric field arise for many electrode geometries and might dictate the interfacial properties. In this context, Galanis and Tsori [9] study the impact of long range electrostatic forces on the demixing behavior of binary dielectric mixtures. We hope that these studies will stimulate further discussions and research directions related to dynamical aspects of electrolytes and their applications.

Finally, we wish to thank again all the contributors for sharing their studies as well as express our gratitude to the editorial members of EPJST for inviting this special issue.

\section{References}

1. W. Dreyer et al., Eur. Phys. J. Special Topics 227, 2515 (2019)

2. B.L. Werkhoven et al., Eur. Phys. J. Special Topics 227, 2539 (2019)

3. M. Matse et al., Eur. Phys. J. Special Topics 227, 2559 (2019)

4. L. Zhang et al., Eur. Phys. J. Special Topics 227, 2575 (2019)

5. S. Bier, A. Yochelis, Eur. Phys. J. Special Topics 227, 2603 (2019)

6. N. Wolff et al., Eur. Phys. J. Special Topics 227, 2617 (2019)

7. M.M. Salman et al., Eur. Phys. J. Special Topics 227, 2641 (2019)

8. Y. Liu, I.Z. Kiss, Eur. Phys. J. Special Topics 227, 2659 (2019)

9. J. Galanis, Y. Tsori, Eur. Phys. J. Special Topics 227, 2675 (2019) 